\title{
EMBEDDINGS INTO THE COMPUTABLY ENUMERABLE DEGREES
}

\author{
M. LERMAN
}

\begin{abstract}
We discuss the status of the problem of characterizing the finite (weak) lattices which can be embedded into the computably enumerable degrees. In particular, we summarize the current status of knowledge about the problem, provide an overview of how to prove these results, discuss directions which have been pursued to try to solve the problem, and present some related open questions.
\end{abstract}

\section{INTRODUCTION}

Degree structures were introduced by Post [25] as structures defined in terms of computability. Because the concept of computability is basic to mathematics, Post expected the study of computability, including the study of degree structures, to be important to mathematics. These structures have since been widely studied from both algebraic and logical viewpoints. Of interest to us is to discover what these structures tell us about the intrinsic nature of computability from oracles. In particular, we would like to know how close the information content of two degrees must be in order for them to look the same within a given degree structure. Cooper [6] has recently announced that there are non-trivial automorphisms of many degree structures, from which it follows that there are degrees with differing information content which look the same inside those degree structures. However, Slaman and Woodin (see [24] for a proof) have shown that if there is an automorphism taking the degree $\mathbf{a}$ to the degree $\mathbf{b}$, then $\mathbf{a}^{\prime \prime}=\mathbf{b}^{\prime \prime}$, so that the information content of $\mathbf{a}$ cannot differ too much from that of $\mathbf{b}$.

The degree structures introduced in Post [25] and in Kleene and Post [9] are defined as follows. Let $A$ and $B$ be sets of natural numbers. We say that $A$ is Turing reducible to $B$ (alternatively, $A$ is computable from $B$ ) and write $A \leq_{T} B$ if there is a computer program with access to $B$ as an oracle which can answer any membership question about $A . \leq_{T}$ is a pre-partial ordering, allowing us to define an equivalence relation $A \equiv_{T} B$ if $A \leq_{T} B$ and $B \leq_{T} A$. The $\equiv_{T}$ equivalence classes are called degrees, and form a partially ordered set under the natural ordering induced by $\leq_{T}$. The corresponding poset $\mathcal{D}=\langle\mathbf{D}, \leq\rangle$ is called the degrees of unsolvability, or the degrees for short. A special subset of $\mathbf{D}$ is the set $\mathbf{R}$ of degrees containing sets which are the output of computer programs with oracle $\emptyset$; we let $\mathcal{R}=\langle\mathbf{R}, \leq\rangle$ denote this poset of computably enumerable (abbreviated as c.e.) degrees.

Date: December 6, 2006.

1991 Mathematics Subject Classification. Primary: 03D25.

Key words and phrases. Embedding, computably enumerable set, computably enumerable degree, lattice.

Research supported by NSF grant DMS-9625445. 
The questions asked in Post [25] and Kleene and Post [9] were algebraic in nature. There were questions about other natural functions and relations which could be defined on the structure (any pair of elements has a join but not necessarily a meet, so the degrees form an upper semi-lattice (usl), and there is a naturally defined jump operator which is order-preserving and takes each degree to a strictly larger degree), and what could be embedded into the structures. Some of these questions have since been more fully answered, and other questions of an algebraic nature such as characterizing extensions of embeddings, homomorphisms, automorphisms and quotients have also been studied. For example, Slaman and Soare [33] have a complete characterization of extensions of embeddings, while Cooper [6] has announced that both $\mathcal{R}$ and $\mathcal{D}$ have non-trivial automorphisms. Other questions of an algebraic nature will be posed in this paper.

Shoenfield [28] was the first to ask types of questions arising from logic about degree structures, conjecturing a characterization of $\mathcal{R}$ which implied that the elementary theory of $\mathcal{R}$ was $\aleph_{0}$-categorical. Shoenfield's conjecture was quickly refuted, but was followed by decidability and undecidability results about degree structures, primarily by Lachlan [11, 12]. However, it was Simpson's [32] characterization of the elementary theory of $\mathcal{D}$ followed quickly by results of Nerode and Shore [22, 23] and Shore [29, 30,31] (among others) which turned the primary focus of the study of degree structures to questions arising from mathematical logic and to global algebraic questions. These questions involved the notions of decidability, definability, and elementary equivalence from mathematical logic, as well as homogeneity and isomorphisms; the latter questions had been raised earlier by Rogers [26]. Simpson called this focus 'global degree theory', and questions of this type motivate much of the current research on degree structures.

Both the algebraic and logical approaches to the study of degree structures have been productive, and the two approaches are closely related. The logical approach introduces constant symbols, relation symbols, and function symbols which determine a language and are to be interpreted over a universe; the algebraic approach begins with a universe, and designates constants, relations and functions which determine the structure. Of course, the symbols in the language of logic interpret the corresponding objects over the universe of study.

We now concentrate on algebraic structures with universe $\mathbf{R}$. There are several choices of language which make sense. We have already introduced the language consisting of the binary relation symbol $\leq$ which gives rise to the structure $\mathcal{R}$. Our main concern will be decidability questions about $T h(\mathcal{R})$, the elementary theory of $\mathcal{R}$, and about fragments of this elementary theory. Harrington and Shelah [8] (see [4] for a proof) showed that $T h(\mathcal{R})$ is undecidable. The fragments of the theory which will be considered will be defined in terms of quantifier complexity. Thus the $\exists$-theory consists of the sentences in prenex normal form, all of whose quantifiers are existential, the $\forall \exists$-theory consists of the sentences in prenex normal form which begin with a block of universal quantifiers, followed by a block of existential quantifiers, followed by a quantifier free formula, etc. Sacks [27] showed that every finite poset can be embedded into $\mathcal{R}$, from which it easily follows that the $\exists$-theory of $\mathcal{R}, \exists \cap T h(\mathcal{R})$, is decidable. Lempp, Nies, and Slaman [15] showed that $\exists \forall \exists \cap T h(\mathcal{R})$ is undecidable. However, it has not yet been determined whether or not the $\forall \exists \cap T h(\mathcal{R})$ is decidable. 
One approach towards studying the decidability of the $\forall \exists \cap T h(\mathcal{R})$ is to look at fragments of this theory which properly extend the $\exists \cap T h(\mathcal{R})$. These are obtained through an algebraic approach; one expands the language by adding naturally and simply defined constant symbols and/or relation symbols and/or function symbols, and studies the existential theory in the expanded language. The most natural choices to add to the language are the constant symbols 0 and 1 denoting the least and greatest elements of $\mathbf{R}$, and the two-place function symbol $\vee$ denoting the least upper bound operator (the least upper bound of two degrees is the degree of the disjoint sum of two sets, one in each degree, which is independent of the choice of sets). This corresponds to studying the c.e. degrees as an upper semi-lattice with least and greatest elements; we denote this structure as $\mathcal{R}_{U, 0,1}$. (The subscripts denote the extra relations, functions, and constants added to the language; we will consider other structures, and the subscripts should make clear the nature of the structure.) Standard techniques easily show, however, that $\exists \cap T h\left(\mathcal{R}_{U, 0,1}\right)$ is decidable. This proof reduces the problem to showing that every finite upper semi-lattice can be embedded into $\mathcal{R}_{U, 0,1}$ preserving least and greatest element if they exist. While lattice structure cannot be placed on $\mathcal{R}$, there are pairs of degrees which have a meet. Thus the next natural step, if one is taking an algebraic approach, is to try to determine which finite lattices can be embedded into $\mathcal{R}$ (as lattices). The natural corresponding logical approach is to introduce predicates $M_{n}\left(a_{1}, \ldots, a_{n}, b\right)$ for all $n \geq 2$ which are defined by the universal formula stating that every element which is $\leq$ every $a_{i}$ is also $\leq b$, and ask about embedding diagrams in the language $\left\langle\leq, \vee,\left\{M_{n}: n \in \mathcal{N}\right\}\right\rangle$, such that the image of the embedding satisfies the defining property of $M_{n}$. We call structures in this language with the natural axioms weak lattices, and the weak lattice with universe $\mathbf{R}$ is denoted as $\mathcal{R}_{W}$. The techniques for studying the embedding questions produced by algebraic and logical approaches are similar, so we will concentrate on the lattice embedding question; in fact, it seems that there are no additional complications, technique-wise, if we ask that the embedding preserve least element, so will study the question asking which finite lattices can be embedded into $\mathcal{R}_{W, 0}$.

There are other predicates which will need to be considered to fully reach $\forall \exists \cap$ $T h(\mathcal{R})$, but we defer discussing these predicates until the end of the paper. We note that instead of working up from the $\exists \cap T h(\mathcal{R})$, one can try to work one's way down from the $\forall \exists \cap T h(\mathcal{R})$. The first natural question which arises from that approach is the algebraic extension of embeddings question. That question was answered by Slaman and Soare [33].

There is an extensive literature dealing with special cases of the lattice embedding problem. We refer the reader to [18] for a more thorough summary than we present here, and will just summarize the major advances from our point of view.

The first lattice embedding result was obtained independently by Lachlan [10] and Yates [37] who embedded $M_{2}$ (see Figure 1), the boolean algebra generated by two atoms, into $\mathcal{R}_{W, 0}$. Those ideas, together with some simple lattice theory, were sufficient to show that all finite (and many infinite) distributive lattices are embeddable into $\mathcal{R}_{W, 0}$. New ideas were needed for the non-distributive case, and were introduced by Lachlan [13] who embedded the two 5-element non-distributive lattices $M_{3}$ and $N_{5}$ (see Figure 1) into $\mathcal{R}_{W, 0}$. These two lattices differ greatly in nature; $N_{5}$ is principally decomposable, i.e., given any two successive elements $b<a$ of the lattice, $\{c: c \leq a \& c \not \leq b\}$ has a minimum, while $M_{3}$ is not 
principally decomposable. (Principally decomposable lattices are also known as lattices without critical triples.) The general belief at that time was that all finite lattice could be embedded into $\mathcal{R}_{W, 0}$. In pursuing embedding strategies, Lerman proposed a lattice, $S_{8}$ (see Figure 2), which could not be embedded using Lachlan's techniques. Subsequently, Lachlan and Soare [14] showed that $S_{8}$ could not be embedded into $\mathcal{R}_{W}$.

$\begin{array}{lll}M_{2} & M_{3} & N_{5}\end{array}$

Figure 1

$S_{8}$

Figure 2

Following the result yielding the non-embeddability of $S_{8}$, Ambos-Spies and Lerman $[2,3]$ tried to understand the embeddability and non-embeddability results. In a series of two papers, they first generalized the Lachlan-Soare non-embeddability result to obtain a non-embedding condition NEC, and then proved a general embeddability theorem. The latter paper introduced a condition EC which was sufficient for embeddability, and left open the question as to whether EC and NEC were complementary conditions. In terms of actual computability-theoretic constructions, there was a technical obstacle which seemed not to be covered by EC or NEC, and the question of complementarity was more a lattice-theoretic question. In 1995, Lempp and Lerman [16] found a (principally decomposable) non-embeddable lattice $L_{20}$ which failed to satisfy EC. Subsequently, in a series of two papers, Lerman $[19,20]$ found a necessary and sufficient condition for embeddability for the class of principally decomposable finite lattices. This condition is not effective - rather it is computable from a $\mathbf{0}^{\prime \prime}$ oracle. We will try to describe this condition in a simple way, and discuss unsuccessful attempts at obtaining an equivalent computable condition and potential directions for further attempts. 


\section{Pinball Machines}

Pinball machines were introduced in [17] to pictorially describe embedding constructions. The numbers which may enter sets, and the sets which these numbers may enter are the labels of balls emanating from holes. These balls roll down a track, and at various stages, are impeded by gates at which they temporarily reside, preventing them from entering a basket (and so having the numbers enter their target sets). New balls may also be introduced at the gates. There are rules governing the release of balls from a hole, and the release of balls by gates, and these rules completely determine the entry of numbers into sets. While the pinball machine for the whole construction is infinite, it suffices to focus on machines with one hole and finitely many gates. Such a machine is pictured below (Figure 3). There are natural rules which govern the action of any pinball machine, and rules which are needed for the satisfaction of requirements. We discuss the former rules in this section, and the latter rules in the next section. The first four rules introduced in this section deal with configurations of the machine, and the remaining three rules deal with transition, i.e., the movement of balls. Fix a pinball machine with hole $H$ and gates $G_{0}, \ldots, G_{n}$.

\section{L14}

Figure 3

The first two rules specify possible ball locations at a given stage.

Rule 1: The game begins with all balls in holes, (so none reside at gates or are in the basket). At all subsequent stages, all balls which have been placed in play either reside at gates or are in the basket.

Rule 2: At the end of the game, all balls placed in play are in the basket.

We define equivalence relations on the balls in play, one for each gate for which the balls placed into play have not yet passed. The relation for $G_{i}$ will collect all balls which are to pass $G_{i}$ simultaneously into the same equivalence class, called a $G_{i}$-block. While this is predictive of the future action of the game, it simplifies some of the later rules. There are two rules governing blocks.

Rule 3: Balls placed in the same $G$-block must reside at the same gate (not necessarily gate $G$ ). 
Rule 4: (a) A ball is placed in a $G_{i}$-block iff it currently resides at gate $G_{j}$ for some $j \geq i$.

(b) All balls which are in the same $G_{i}$ block (where $i<n$ ) and do not currently reside at $G_{i}$ must be in the same $G_{i+1}$-block.

The next set of rules are transition rules which govern the movement of balls between successive configurations. The first of these rules tells us which balls move, and their next location. The second rule tells us that the order of balls is preserved, and the movement from gate to gate respects the ordering of the gates. The third rule prohibits blocks from changing when we pass to a new configuration.

Rule 5: Fix the smallest $i$ such that $G_{i}$ is currently inhabited. The balls in play for the current configurations which change location for the next configuration are precisely the balls which are in the first $G_{i}$-block of $G_{i}$, and these move to $G_{i-1}$ if $i>0$ and to the basket if $i=0$.

Rule 6: Balls not entering the basket between configurations maintain their order.

Rule 7: (a) Balls which are in the same $G_{i}$-block for the current configuration and reside at gate $G_{j}$ for some $j \geq i$ for the next configuration remain in the same $G_{i}$-block for the next configuration.

(b) Balls currently in play which are not in the same $G_{i}$-block for the current configuration cannot be in the same $G_{i}$-block for the next configuration.

\section{Rules FOR REQuirements}

We now consider requirements for the embedding construction, and impose rules on the pinball machine game which, if obeyed, will ensure the satisfaction of the requirements. Suppose that $\langle L, \leq, \vee, \wedge\rangle$ is a finite lattice which we wish to embed into $\mathcal{R}_{W, 0}$ preserving join and meet. We will use lower-case letters for elements of $L$, and the corresponding upper-case letters will denote the sets of integers whose degrees characterize the embedding into $\mathcal{R}_{W, 0}$.

The first type of embedding requirement will ensure that order is preserved. Thus if $a<b \in L$, we wish to ensure that $A \leq_{T} B$. We do so by requiring that if a ball labeled with target $A$ and number $x$ enters the basket, then $x$ is placed into all sets $C$ such that $A \leq_{T} C$. This is enough to ensure the satisfaction of the order-preserving requirements, and does not require the imposition of any rules on the game.

The second type of embedding requirement will ensure that incomparability is preserved. Thus if $a \not \leq b \in L$, we wish to ensure that $A \not_{T} B$. The following rule will ensure the satisfaction of this requirement.

Rule 8: If $a \not \leq b$, then the initial configuration has a ball with target $\leq_{T} A$, but no ball with target $\leq_{T} B$.

The third type of embedding requirement will ensure that joins are preserved. Suppose that $a \vee b=c \in L$. As $a, b \leq c$, the satisfaction of the order-preserving requirements will ensure that $A \oplus B \leq_{T} C$. Thus it suffices to ensure that $C \leq_{T}$ $A \oplus B$. The standard way to satisfy such a requirement is to use the method of traces. This method ensures that when a number $x$ is targeted for $C$, then another number $y$ is targeted for $A$ or $B$, and $y$ must enter its target set at a stage $s$ which is no later than the stage at which $x$ enters $C$; furthermore, if $x$ does not enter $C$ 
at stage $s$, then a new trace $z$ for $x$ is appointed at stage $s$, and is burdened with the same restrictions as was $y$. The success of this method relies on showing that any number $x$ has only finitely many traces during the course of the construction. The following rule corresponds to the method of traces.

Rule 9: If $a \vee b=c$, then for every configuration, if a ball has target $\leq_{T} C$, then there is a predecessor ball whose target is either $\leq_{T} A$ or $\leq_{T} B$.

The final type of embedding requirement will ensure that meets are preserved. Suppose that $a \wedge b=c \in L$. It will follow from the order-preserving requirements that $C \leq_{T} A, B$. Thus we must ensure that for every c.e. set $U$, if $U \leq A, B$ then $U \leq_{T} C$. In fact, we need not restrict our attention to c.e. sets. Thus for each pair of computable partial functionals $\Phi$ and $\Psi$, we construct a computable partial functional $\Delta$ such that whenever $\Phi(A)=\Psi(B)$ and $\Phi(A)$ is total, then $\Delta(C)=\Phi(A)$. The strategy is to define $\Delta(C ; x)[s+1]=\Phi(A ; x)[s]$ whenever $\Phi(A ; y)[s]=\Psi(B ; y)[s]$ for all $y \leq x$, and then to allow numbers to enter only $A$ or only $B$ to injure the equality until the equality recovers, unless a number which injures the axiom $\Delta(C ; x)[s+1]$ enters $C$ in the interim. Thus at all stages $t+1$ at which $\Delta(C ; x)[t+1] \downarrow$, either $\Delta(C ; x)[t+1]=\Phi(A ; x)[s]$ or $\Delta(C ; x)[t+1]=$ $\Psi(B ; x)[s]$. The implementation of this condition as a pinball machine rule requires the introduction of the new notion of a $G$-block being $G$-prohibited. We will define this notion after presenting the rule, but indicate the intuition behind the notion now; if $G$ corresponds to $p \wedge q=r$, then a $G$-block is $G$-prohibited if the introduction of a new ball with target $\leq r$ into the block and its later entry into the basket will cause injury to the computations from both $P$ and $Q$ on some argument $x$, without correcting the computation from $R$. The following rule formalizes $G_{i}$-prohibitions for $i \leq n$.

Rule 10: If a $G_{i}$-block is released by gate $G_{i}$, then at the time of release the block is not $G_{i}$-prohibited.

Before defining prohibitions, we present two examples to provide some intuition.

Example 1: Consider the lattice $N_{5}$ pictured in Figure 1. The incomparability requirement $C \Varangle_{T} A$ requires the placement of a ball with target $C$ on the pinball machine, and as $a \vee b \geq c$, Rule 9 requires this ball to be preceded by a ball with target $B$ or $A$; as Rule 8 precludes target $A$, our first sequence will be $\langle B, C\rangle$. The meet condition $c \wedge b=0$ at gate $G$ requires that the two balls with targets $B$ and $C$ respectively be in different blocks, else the simultaneous entry of both balls into their respective target sets would injure the requirement (thus the single block would have a $G$-prohibition). The block consisting of a single ball with target $B$ comes first, and the block consisting of a single ball with target $C$ has a $G$ prohibition. Once the ball with target $B$ enters the basket, a new ball with target $A$ is appointed, and placed in the same $G$-block as the ball with target $C$, and the $G$-prohibition is removed. At this point, the simultaneous entry of these two balls into the basket will give rise to a target sequence $\langle A, C\rangle$, and entry of balls into these sets cannot injure a computation from oracle $B$, so the requirement will not be injured. 
Figure 4

Example 2: Consider the lattice $L_{14}$ of Figure 4 . We first note that $a \vee b \geq f, d$ and $d \vee e \geq f$. Thus in order to satisfy the incomparability requirement corresponding to $f \not \leq e$, we may assume by symmetry that our starting array on the pinball machine has balls targeted, in order, for $A, D$, and $F$. Let the meet requirement $(f \vee c) \wedge d=0$ be assigned to gate $G$, the first gate that the above sequence of balls will encounter when released from the hole. As $a, f \leq f \vee c$, these balls must all be in different $G$-blocks else the meet requirement will be injured. When the first ball enters its target set, a suitable next configuration of the pinball machine would have balls at gate $G$ targeted, in order, for $B, D, A$, and $F$, where the first and third balls are new, and the second and fourth balls are old. Furthermore, we can place the first two balls in one $G$-block, and the last two balls in another $G$-block. Now suppose that the first two balls enter the basket simultaneously. A possible next sequence would have three balls targeted, in order, for $A, E$, and $F$, where the middle ball is a new ball and the other two balls are old balls. All three balls may be placed in the same $G$-block, and so will be released simultaneously by gate G.

The above strategy will work if $G$ is the only gate. Suppose now that there is a second gate $G^{\prime}$ which corresponds to the meet requirement $(b \vee e) \wedge(a \vee e)=e$. Consider the $G^{\prime}$-blocks for the configuration with targets $B, D, A, F$. The $G^{\prime}$-blocks containing $A$ and $F$ must have $E$-prohibitions, as a new ball targeted for $E$ will be appointed in a location where computations from $A \oplus E$ and $B \oplus E$ will be injured but the new $E$ ball has a number too large to correct the computation from the meet. In this situation, we can pass to a configuration with four balls targeted, in order, for $A, E, A$, and $F$, where the first two balls are new balls and the last two balls are old balls, and place all the balls in the same $G$-block but the second and third balls in different $G^{\prime}$-blocks, and will be able to successfully continue the game when the balls are simultaneously released by $G$ and roll to $G^{\prime}$.

We now present the three gate prohibition rules. The first rule governs the removal of old $G$-prohibitions, and the last two rules govern the imposition of new $G$-prohibitions, one rule for old $G$-blocks and one rule for new $G$-blocks. Let $B$ be a $G$-block of the current configuration. Suppose that $p \wedge q=r$ is the meet requirement assigned to gate $G$, and let $K$ be the upward-closure of the lattice 
elements representing targets for the balls in the $G$-blocks preceding $B$ or in $B$ itself.

Rule 11: If $B$ is an old $G$-block (a $G$-block containing balls from the preceding configuration) and the block of the preceding configuration which is extended by $B$ has a $G$-prohibition, then this $G$-prohibition is removed if and only if the first inhabited gate of the current configuration is either gate $G$ or a gate above gate $G$, and either $p \notin K$ or $q \notin K$.

Rule 12: If the first inhabited gate is above gate $G$, then a new $G$-prohibition is imposed on block $B$ if and only if $p, q \in K$ but $r \notin K$.

Rule 13: If the first inhabited gate is either gate $G$ or a gate below gate $G$, then a new $G$-prohibition is imposed on block $B$ if and only if $B$ is a new block (i.e., $B$ contains no balls from the immediately preceding configuration) and the last old $G$-block which precedes $B$ on the current configuration of the pinball machine has a $G$-prohibition in the immediately preceding configuration which is not removed by Rule 11 .

A necessary and sufficient condition, GBTA, for embedding principally decomposable finite lattices into the c.e. degrees is presented in Lerman [20], and is rephrased here in terms of the rules for games.

Definition 3.1. GBTA: For every pinball machine game as above (one hole and a finite sequence of gates) there is a finite array of configurations for that pinball machine game which satisfies Rules 1-13. (Such an array is called a good blocked target array.)

Note that $G B T A$ is an $\forall \exists$ condition, so can be decided by an oracle of degree $\mathbf{0}^{\prime \prime}$. The sufficiency of $G B T A$ is proved by a standard embedding construction (pinball machine model), noting that if the condition is true, then a finite search will effectively produce the sequence of configurations, given the game; (i.e., if an $\forall \exists$ sentence has a Skolem function, then it has a computable Skolem function). The necessity of GBTA is obtained by reversing the portion of the embedding construction corresponding to any choice of a hole and a finite sequence of gates, and showing that the failure of the opponent to win such a game can be translated into a sequence of configurations for that game which satisfies Rules 1-13. The restriction to principally decomposable lattices is needed for the non-embedding games, as we must ensure that we can always get by with complete trace functions for join requirements that are finite at every stage. For ranked lattices (defined in Lerman [19], all tracing functions are finite. In [20], it is shown that, while not all complete trace functions are finite at every stage if the lattice is not ranked, one can replace the trace function at any given stage with a complete finite trace function. If the lattice is not principally decomposable, then there is no way to

avoid using infinite complete trace functions, and the non-embedding game cannot be implemented.

\section{Attempts to Determine Whether $G B T A$ is Equivalent to AN EFFECTIVE CONDITION}

Several approaches have been tried to show that $G B T A$ is equivalent to an effective condition. These include: 
- Trying to approximate to a given lattice through smaller subsets of the lattice, and to find a relationship between the configurations for the smaller subsets and the configurations for the lattice itself. As subsets, we have looked at sublattices, subsets which preserve upper semi-lattice operations, and, most atomically, we have looked at configurations obtained by adding one requirement at a time. In all cases, we were unable to find a uniform way of extending configurations for the subset to configurations for the larger set of requirements which either produces an obstruction implying non-embeddability, or produces a sequence of configurations satisfying all rules for the larger set of requirements.

- Defining a concept of normal form for configurations, and showing that we can effectively compute, for each game, a bound on the length of the normal form sequences which are relevant to that game. The first attempt to define a normal form was to try to use sequences which satisfied the tracing requirements and were minimal in this regard. Such sequences place a restriction on the ability of meet requirements to correct computations, and we later found an example of a sequence of requirements which could be satisfied, but could not be satisfied with this proposed definition of normal form. We then turned unsuccessfully to sequences without repetitions, and, having failed to define a suitable notion of normal form, to seeing if there was a uniform way of piecing together uniformly bounded configurations to obtain a full configuration. These attempts were also unsuccessful. Both Nerode, and later Khoussainov, suggested that the problem might be coded into the decision procedure for $S 2 S$; but the only way we saw to do approach this required us to first show that there is a bound on the size of configurations for a given game, so we encounter the same obstacle. The basic problem, even for a single game, is that the rules for prohibition functions are not local (restricted to a small interval of the configuration) rules.

Shore has noted that the methods used to code in an undecidable fragment of a theory cannot be used to obtain undecidability results; rather, one must directly try to code the halting problem, the word problem for groups, or some other basic undecidability result. These undecidability results involve analogs of sequences of configurations, but changes in passing from configuration to configuration are local, while the pinball machine game configurations are not, making it difficult to see how such a coding might go. As non-local conditions seem to be more complicated than local conditions, this might be taken as evidence for an intuitive guess that the lattice embedding problem is undecidable.

\section{Open Questions Related to Embeddings}

As was mentioned in the introduction, one can approach the study of $\mathcal{R}$ by asking the questions a logician would ask, or by asking the questions an algebraist would ask. From a logician's point of view, the main open question is:

Question 1: Is $\forall \exists \cap T h(\mathcal{R})$ decidable?

A suggested approach was to enrich the language, and to try to decide the $\exists$ theory in a richer language. This gave rise to the notion of weak lattice, and the question:

Question 2: Is there an algorithm to determine which finite weak lattices are embeddable into $\mathcal{R}_{W}$. 
As the techniques to embed lattices and weak lattices seem to be identical, and as we are more familiar with lattices, we are led to the question which this paper has focused on, namely:

Question 3: Is there an algorithm to determine which finite lattices are embeddable into $\mathcal{R}_{W}$.

The pursuit of a negative answer to Question 1 might proceed by trying to code an undecidable problem into the existential theory of a structure obtained by enriching the language. For as we come closer to the full $\forall \exists \cap T h(\mathcal{R})$ and find necessary and sufficient conditions for the corresponding decision problems, we should expect more flexibility in our ability to code undecidable problems into these necessary and sufficient conditions. Thus it is reasonable to further extend the language, and to try to find a condition which characterizes the decision problem for the existential theory of the enriched structure. Lerman [18] has shown that $G B T A$ can be converted to a necessary and sufficient condition for an existential sentence of $\mathcal{R}_{W}$ to be true, and this condition remains unchanged for $\mathcal{R}_{W, 0}$. Englert (work in progress) has a similar result for $\mathcal{R}_{W, 1}$

Question 4: Is there an algorithm to determine which finite weak lattices are embeddable into $\mathcal{R}_{W, 0,1}$ ?

Similar questions can be asked for other enriched languages whose $\exists$-theory is a subset of the $\forall \exists \cap T h(\mathcal{R})$. Predicates of this type were crucial in the extension of embeddings result of Slaman and Soare [33].

The methods described in this paper can be used to study embeddings into structures other than $\mathcal{R}$. We mention two problems of this type which have been studied. The first is the problem of effectively characterizing the finite (weak) lattices which can be embedded into every non-trivial initial segment of $\mathcal{R}$, i.e., every interval of the form $[\mathbf{0}, \mathbf{a}]$ where $\mathbf{a} \neq \mathbf{0}$ is a c.e. degree. Weinstein [36] and Downey [7] showed that every such (weak) lattice must be principally decomposable, and Lerman [20] noted that a principally decomposable (weak) lattice $L$ is embeddable into every non-trivial initial segment of $\mathcal{R}$ iff $L$ satisfies $G B T A$. Thus the problem again reduces to the question of whether $G B T A$ is equivalent to an effective condition.

The other structure is that of the upper semi-lattice $\mathcal{I}$ of ideals of c.e. degrees, which has been studied by Calhoun [5]. Calhoun showed that the lattice $S_{8}$ (which Lachlan and Soare showed could not be embedded into the c.e. degrees) is embeddable into $\mathcal{I}$. Thus the obstruction to embedding witnessed by $S_{8}$ is not an obstruction for $\mathcal{I}$. However, the obstruction to embedding witnessed by the lattice $L_{20}$ (which Lempp and Lerman showed could not be embedded into the c.e. degrees) still seems to remain, and Calhoun and Lerman have a preliminary sketch which indicates that $L_{20}$ cannot be embedded into $\mathcal{I}$. In fact, they feel that it is highly likely that a principally decomposable finite (weak) lattice is embeddable into $\mathcal{I}$ iff it satisfies $G B T A$.

\section{Open Questions: Homomorphisms}

We discussed the algebraic approach to studying degree structures in the Introduction. One topic which falls under this heading and has not received much attention is that of homomorphic images of degree structures (with the exception of 
automorphism questions) and homomorphisms and endomorhisms of degree structures. We will focus primarily on $\mathcal{R}$, but note that similar questions can be asked about all degree structures.

We begin by trying to determine whether a degree structure has any non-trivial homomorphic images, i.e., whether the structure is simple. The definition of simplicity is meant to rule out all but the trivial homomorphic images, i.e., the maps onto the structure itself, and the maps onto a single element.

Definition 6.1. A degree structure $\mathcal{S}$ with universe $S$ is simple if for every homomorphism $f$ with domain $S$, either $f(S)=S$ or $|f(S)|=1$.

Question 5: Which degree structures are simple?

The map $j^{n}$ taking a degree to its $n t h$ jump is a poset homomorphism. Shore $[\mathrm{S}]$ has shown that if $n \geq 3$, then the original degree structure and its homomorphic image are not isomorphic. Furthermore, any such map on the standard degree structures has infinite range. Hence the standard degree structures, viewed as posets, are not simple. A substantial literature, due to Martin [21], Steel [35], and Slaman-Steel [34] exists studying poset homomorphisms on degree structures which take every degree $\mathbf{a}$ to a degree $\mathbf{b} \geq \mathbf{a}$. Such homomorphisms, however, are not upper semi-lattice homomorphisms.

The only investigation of upper semi-lattice homomorphisms of which we are aware is for the upper semilattice $\mathcal{R}_{U}$ of c.e. degrees. Ambos-Spies, Jockusch, Shore and Soare [1] showed that the cappable degrees form an ideal $\mathbf{M}$ whose complement PS is a strong filter. Thus we can define a pre-order relation $\cong_{M}$ on $\mathbf{R}$, setting $\mathbf{a} \leq_{\mathbf{M}} \mathbf{b}$ if there is a cappable degree $\mathbf{c}$ such that $\mathbf{a} \leq \mathbf{b} \cup \mathbf{c}$. We then define $\mathbf{a} \cong_{\mathbf{M}} \mathbf{b}$ iff $\mathbf{a} \leq_{\mathbf{M}} \mathbf{b}$ and $\mathbf{b} \leq_{\mathbf{M}} \mathbf{a}$, and note that the map taking a degree in $\mathbf{R}$ to its congruence class in $\mathbf{R} / \mathbf{M}$ is a usl homomorphism. Thus $\mathcal{R}_{\mathcal{U}}$ is not simple, as $\mathbf{0}$ and $\mathbf{0}^{\prime}$ are in different congruence classes. $\mathbf{R} / \mathbf{P S}$ is similarly defined. Leonhardi, Lerman and Yi (unpublished) have shown that the above homomorphism from $\mathbf{R}$ to $\mathbf{R} / \mathbf{M}$ is not a weak lattice homomorphism. However, the map taking $\mathbf{M}$ to 0 and PS to 1 is a weak lattice homomorphism of $\mathcal{R}_{W}$, so $\mathcal{R}_{W}$ is also not simple.

An old question, mentioned in both the talks of Nies and Shore at this conference, is whether there is any other definable ideal of $\mathcal{R}$ whose complement is a strong filter. This is part of the more general question:

Question 6: Characterize all weak lattice homomorphisms of $\mathcal{R}$ onto $\{0,1\}$.

Other related questions, asked for $\mathcal{R}$ but appropriate for other degree structures are:

Question 7: Characterize all homomorphic images (quotients) of $\mathcal{R}$.

Question 8: Characterize all (definable) homomorphisms of $\mathcal{R}$.

We conclude with an observation of Lempp that there is no usl homomorphism mapping the d-c.e. degrees onto the c.e. degrees. This follows easily from the fact that every d-c.e. degree can be cupped to $\mathbf{0}^{\prime}$, while not every c.e. degree can be cupped to $\mathbf{0}^{\prime}$.

\section{REFERENCES}

[1] K. Ambos-Spies, C. G. Jockusch, R. A. Shore, and R. I. Soare, An algebraic decomposition of the recursively enumerable degrees and the coincidence of several degree classes with the promptly simple degrees, Trans. Amer. Math. Soc. 281 (1984), 109-128. 
[2] K. Ambos-Spies and M. Lerman, Lattice embeddings into the recursively enumerable degrees, J. Symbolic Logic 51 (1986), 257-272.

[3] K. Ambos-Spies and M. Lerman, Lattice embeddings into the recursively enumerable degrees: II, J. Symbolic Logic 54 (1989), 735-760.

[4] K. Ambos-Spies and R. A. Shore, Undecidability and 1-types in the recursively enumerable degrees, Ann. Pure Appl. Logic 63 (1993), 3-37.

[5] W. C. Calhoun, The lattice of ideals of recursively enumerable degrees, Ph.D. Dissertation, University of California at Berkeley, 1990.

[6] S. B. Cooper, The Turing universe is not rigid, to appear.

[7] R. G. Downey, Lattice non-embeddings and initial segments of the recursively enumerable degrees, Ann. Pure Appl. Logic 49 (1990), 97-119.

[8] L. Harrington and S. Shelah, The undecidability of the recursively enumerable degrees (research announcement), Bull. Amer. Math. Soc. (N. S.) 6 (1982), 79-80.

[9] S. C. Kleene and E. L. Post, The upper semi-lattice of degrees of recursive unsolvability, Ann. Math. (2) 59 (1954), 379-407.

[10] A. H. Lachlan, Lower bounds for pairs of recursively enumerable degrees, Proc. London Math. Soc. 16 (1966), 537-569.

[11] A. H. Lachlan, Distributive initial segments of the degrees of unsolvability, Z. Math. Logik Grund. Math. 14 (1968), 457-472.

[12] A. H. Lachlan, The elementary theory of recursively enumerable sets, Duke Math Jour. 35 (1968), 123-146.

[13] A. H. Lachlan, Embedding nondistributive lattices in the recursively enumerable degrees, In: Conference in Mathematical Logic, 1970, W. Hodges ed., Lecture Notes in Mathematics 255, Springer-Verlag, Berlin, Heidelberg, New York, 1972, 149-177.

[14] A. H. Lachlan and R. I. Soare, Not every finite lattice is embeddable in the recursively enumerable degrees, Advances in Math. 37 (1980), 74-82.

[15] S. Lempp, A. Nies and T. A. Slaman, The $\Pi_{3}$-theory of the enumerable Turing degrees is undecidable, Trans. Amer. Math. Soc. 350 (1998), 2719-2736.

[16] S. Lempp and M. Lerman, A finite lattice without critical triple that cannot be embedded into the enumerable Turing degrees, Ann. Pure Appl. Logic 87 (1997), 167-185.

[17] M. Lerman, Admissible ordinals and priority arguments, In: Cambridge Summer School in Mathematical Logic - Proceedings 1971, A. R. D. Mathias and H. Rogers eds., Lecture Notes in Mathematics, 337, Springer-Verlag Pub. Co., Berlin, Heidelberg, New York, 1973, 311-344.

[18] M. Lerman, Embeddings into the recursively enumerable degrees, In: Computability, Enumerability, Unsolvability: Directions in Recursion Theory, S. B. Cooper T. A. Slaman and S. S. Wainer eds., London Math. Soc. Lecture Note Series, 224, Cambridge University Press, Cambridge, 1996, 185-204.

[19] M. Lerman, A necessary and sufficient condition for embedding ranked finite partial lattices into the computably enumerable degrees, Ann. Pure Appl. Logic 94 (1998), 143-180.

[20] M. Lerman, A necessary and sufficient condition for embedding principally decomposable finite partial lattices into the computably enumerable degrees, Ann. Pure Appl. Logic, to appear.

[21] D. A. Martin, The axiom of determinateness and reduction principle in the analytical hierarchy, Bill. Amer. Math. Soc. 74 (1968), 687-689.

[22] A. Nerode and R. A. Shore, Second order logic and first order theories of reducibility orderings, In: The Kleene Symposium, K. J. Barwise, H. J. Keisler, and K. Kunen eds., NorthHolland Pub. Co., Amsterdam, New York, Oxford, 1980, 181-200.

[23] A. Nerode and R. A. Shore, Reducibility Orderings: Theories, definability and automorphisms, Ann. Math. Logic 18 (1980), 61-89.

[24] A. Nies, R. A. Shore, and T. A. Slaman, Interpretability and definability in the recursively enumerable degrees, Proc. London Math. Soc. (3) 77 (1998), 241-291.

[25] E. L. Post, Recursively enumerable sets of positive integers and their decision problems, Bull. Amer. Math. Soc. 50 (1944), 284-316.

[26] H. Rogers, Jr., Theory of Recursive Functions and Effective Computability, McGraw-Hill, New York, 1967.

[27] G. E. Sacks, Degrees of Unsolvability, Annals of Mathematics Studies, 55, Princeton Univ. Press, Princeton, 1963. 
[28] J. R. Shoenfield, Application of Model Theory to degrees of unsolvability, In: Symposium on the Theory of Models, J. W. Addison, L. Henkin and A. Tarski eds., North-Holland Pub. Co., Amsterdam, 1965, 359-363.

[29] R. A. Shore, The homogeneity conjecture. Proc. Nat. Acad. Sci. U. S. A. 76 (1979), 42184219.

[30] R. A. Shore, The theory of the degrees below 0', J. London Math. Soc. (2) 24 (1981), 1-14.

[31] R. A. Shore, On homogeneity and definability in the first-order theory of the Turing degrees. Jour. Symb. Logic 47 (1982), 8-16.

[32] S. G. Simpson, First-order theory of the degrees of recursive unsolvability, Ann. Math. (2) 105 (1977), 121-139.

[33] T. A. Slaman and R. I. Soare, Algebraic aspects of the computably enumerable degrees, Proc. Nat. Acad. Sci. USA 92 (1995), 617-621.

[34] T. A. Slaman and J. R. Steel, Definable functions on degrees,

[35] J. R. Steel, A classification of jump operators, Jour. Symb. Logic 47 (1982), 347-358.

[36] B. Weinstein, On embeddings of the 1-3-1 lattice into the recursively enumerable degrees, Doctoral Dissertation, University of California at Berkeley, 1988.

[37] C. E. M. Yates, A minimal pair of recursively enumerable degrees, Jour. Symb. Logic 31 (1966), 159-168.

Department of Mathematics, University of Connecticut, Storrs, CT 06269-3009 USA

E-mail address: mlerman@math.uconn.edu 